\title{
Reporte de caso: fístula carótido-cavernosa post-traumática en un adolescente
}

\section{Case report: post-traumatic carotid-cavernous fistula in a teenager}

\section{Pág. 107,114}

Recibido: 28-04-2020

Aceptado: $26-05-2020$

Dra. Constanza Chacón González. Investigadora independiente, Médico general , San José, Costa Rica

Dr. Ariel Mendelewicz Montero. Investigador independiente, Médico general , San José, Costa Rica

Dra. Luisana Guier Bonilla. Investigadora independiente, Médico general , San José, Costa Rica

\section{Resumen}

Las FCCs son comunicaciones vasculares entre el sistema arterial carotídeo y el sistema de drenaje venoso cerebral; principalmente el seno cavernoso.

A pesar de haber sido de las primeras lesiones vasculares intracerebrales en describirse, su poca ocurrencia dificulta la realización de grandes estudios que estandarice su abordaje y manejo. El objetivo de este caso clínico es describir una FCC diagnosticada en el Hospital México, de la Caja Costarricense de Seguro Social (CCSS) en San José Costa Rica, y de esta forma contribuir a la literatura médico-científica.

Se reporta el caso de un paciente masculino de 17 años víctima de un poli-trauma, su sintomatología, el abordaje diagnóstico y la intervención terapéutica. A su vez se realiza una revisión del tema que evidencia la unicidad y variabilidad de esta patología, y se concluye que es indispensable la sospecha clínica y pronto manejo para así disminuir su morbilidad.

Palabras claves: Seno cavernoso, fístula, proptosis, oftalmoplegia, exoftalmia pulsá-til, tratamiento endovascular.

\section{Abstract}

Carotid-cavernous fistulas (CCF) are vascular communications between the ca-rotid arteries and the cerebral venous drainage system; mainly the cavernous sinus. Despite being one of the first intracerebral vascular injuries ever described, its rarity makes it difficult to carry out large studies to standardize its approach and manage-ment.

The purpose of this case report is to describe a CCF diagnosed at Hospital Mex-ico at the Caja Costarricense de Seguro Social (CCSS) in San José, Costa Rica, and make a contribution to the medical community

The case of a 17 year old male victim of multiple trauma is reported, along with his symptoms, the diagnostic approach, and therapeutic intervention. At the same time, a review of the subject sheds light to the uniqueness of this pathology, and it is con-cluded that it is of utmost importance to suspect this diagnosis and approach it prompt-ly in order to reduce morbidity.

Key words: Cavernous sinus, fistula, proptosis, ophthalmoplegia, pulsating exoph-thalmos, endovascular treatment. 


\section{Introducción}

La fístula carótida-cavernosa (FCC) es una patología inhabitual que implica la comunicación entre la arteria carótida interna (ACl), arteria carótida externa (ACE), alguna de sus ramas, y el seno cavernoso $(1,2)$. Anomalías en la vascularidad carotí-dea se empezaron a documentar desde principios del siglo XIX cuando médicos eu-ropeos notaron exoftalmos pulsátiles en distintos pacientes (2). Sin embargo, no fue hasta 1935 cuando Walter Edward Dandy descubrió conexiones arteriovenosas anó-malas en autopsias de pacientes con características clínicas de FCC (2).

Dicha condición puede ser iatrogénica, secundaria a trauma, o bien se puede dar de manera espontánea $(1,3)$. Se pueden clasificar según la región anatómica afectada, las características hemodinámicas, o su origen $(4,5)$. La FCC es de difícil diagnóstico, requiere un alto grado de sospecha y el conocimiento de sus múltiples manifestaciones clínicas $(1,6)$.Si no se trata prontamente pueden ocurrir complicacio-nes y secuelas tan severas comola ceguera permanente. La literatura a nivel mundial describe casos clínicos, pero dado su unicidad no existen grandes estudios recientes para estandarizar su abordaje y tratamiento. En las últimas dos décadas se han publicado sólo dos revisiones de tema en Costa Rica $(7,8)$.

El objetivo de este artículo es reportar el caso de un paciente de 17 años al que se le diagnosticó una FCC en el Hospital México de la Caja Costarricense del Seguro Social (CCSS) en San José, Costa Rica, y compartir el abordaje de un caso único con la comunidad médica nacional y latinoamericana.

\section{Reporte de caso}

Un paciente masculino de 17 años fue trasladado al Hospital México desde el Hospital Enrique Baltodano de Liberia con historia de haber sido atropellado por un carro y sufrir múltiples traumas contusos en cráneo.

A la valoración en sala de shock se evidenció estigmas de trauma en cara. Se solicitó una tomografía axial computarizada (TAC) de cráneo y macizo facial con re-construcción 3D. EI TAC mostró una fractura parasinfisiaria mandibular derecha, frac-tura de cóndilo mandibular derecho y fractura malar y de arco cigomático derecho, por lo que fue ingresado al servicio de cirugía plástica. Las figuras 1 y 2 muestran dos cor-tes del TAC de macizo facial evidenciando las fracturas.

El paciente fue llevado a sala de operaciones cuatro días después de su pre-sentación inicial para reducción abierta de sus fracturas con colocación de placas de fijación interna y fijación intermaxilar transoperatoria. El procedimiento transcurrió sin complicaciones. En el día 1 postoperatorio el examen físico no demostró datos de infección, la herida estaba sin secreciones o hematomas, las suturas estaban íntegras y había edema leve en la zona periorbitaria y mandibular derecha; esperable para la intervención que se le realizó. El paciente fue dado de alta ese mismo día.

El paciente se presentó a su cita de curación de heridas siete días tras el alta hospitalaria. La exploración evidenció marcado edema palpebral con restricción de la apertura ocular y proptosis, demostrado en la figura 3 , asociando disminución de la agudeza visual en el ojo derecho. El paciente fue derivado al servicio de emergencias para realización de TAC por sospecha de un posible síndrome de atrapamiento ocu-lar o síndrome compartimental de órbita.

Nuevas imágenes de TAC, demostradas en la figura 4, documentaron fracturas del hueso etmoides y esfenoides manejadas de forma conservadora por recomenda-ción del servicio 
de otorrinolaringología. Se descartó patología neuroquirúrgica.

El examen oftalmológico indicó disminución de la agudeza visual, edema pe-riorbitario, equimosis, y proptosis que se manejó con acetazolamida. Se ingresó para completar estudios vigilando evolución por riesgo de síndrome compartimental orbita-rio. Se iniciaron esteroides para disminuir la inflamación.

Ante la persistencia del edema y la ausencia de respuesta a dosis altas de es-teroides se decide iniciar cobertura antibiótica empírica con vancomicina durante tres días. En una valoración oftalmológica subsecuente sobresale el hallazgo de pulsa-ciones a nivel del párpado superior derecho. Esto conlleva a la sospecha de una FCC, por lo que se sugiere completar imágenes para confirmar este diagnóstico.

Se realizó un TAC de sistema nervioso central (SNC) con medio de contraste que describió hallazgos sugestivos de una FCC: proptosis de globo ocular derecho con desplazamiento $28 \mathrm{~mm}$ de borde anterior respecto a línea intercantal (lo normal es $<20 \mathrm{~mm}$ ), a correlacionar con estudio angiográfico. La resonancia magnética (RM) describe una dilatación de la vena oftálmica superior derecha, cambios inflamatorios de la grasa retroocular derecha y cambios de intensidad en señal del flujo hacia el seno cavernoso.

Enseguida se realizó una arteriografía diagnóstica y terapéutica con técnica Seldinger. Se hizo una arteriotomía femoral derecha hacia carótidas comunes, $\mathrm{ACl}, \mathrm{ACE}$ y arterias vertebrales. Las imágenes demostraron la presencia de una FCC de-recha, ectasia de la arteria oftálmica superior derecha, senos petrosos superior e inferior derecho, seno cavernoso derecho y plexo pterigopalatino. Se observó escaso lle-nado de la $\mathrm{ACl}$ supraclinoidea derecha, con flujo proveniente de la $\mathrm{ACl}$ izquierda, su-gestivo de adecuada irrigación colateral.

Previo comprobación de adecuada perfusión cerebral hacia el hemisferio derecho a través de las arterias comunicantes anterior y posterior, se realizó una cateterización supraselectiva de seno cavernoso embolizando con un coil y ocluyendo la $\mathrm{ACl}$ derecha.

El paciente se egresó sin complicaciones. En una cita control, 2 meses poste-rior al accidente, se observó mejoría sustancial de su síntomas, como se evidencia en la figura 5. A la fecha, habiendo transcurrido 2 años desde la intervención, no se han documentado secuelas.

\section{Discusión}

La comunicación anormal entre el seno cavernoso y el sistema carotídeo se denomina FCC (9). Dicha condición requiere de alta sospecha clínica, diagnóstico oportuno y un tratamiento específico, por lo que es importante tener clara la anatomía de la región con el fin de poder explicar sus manifestaciones clínicas. (10)

El seno cavernoso es un plexo venoso trabecular extradural. Se comunica a través de venas emisarias con la fisura orbitaria superior, a través del foramen redon-do con la fosa pterigopalatina, a través del foramen oval con la región pterigoidea y a través del seno petroso superior con la vena yugular interna. $(4,11)$

Es una región con múltiples estructuras vasculonerviosas, adyacente a la silla turca y en íntimo contacto con la $\mathrm{ACl}$ bilateralmente. La pared lateral del seno contie-ne los nervios oculomotor, troclear, primera y segunda división del trigémino, y abdu-cens. El seno cavernoso drena las venas oftálmicas superior e inferior y el seno esfe-noparietal, para así drenar a través del seno petroso superior e inferior hacia el seno yugular, el plexo pterigoideo y al seno cavernoso

\section{(9) $(1) \Theta \theta$}


contralateral. Cuando se crea una fístula, se forma una comunicación arteriovenosa que resulta en una alteración en el flujo sanguíneo normal de la zona. $(4,11)$

Las FCC se pueden clasificar según sus características hemodinámicas (alto o bajo flujo), por su etiología (traumática o espontánea), y por su anatomía, que es la que más comúnmente se usa y fue descrita por Barrow $(1,4,5,9,11,12$, $13,14,15)$. La clasificación de Barrow divide las FCC en directas (tipo A) e indirectas (tipos B, C, D) según donde se encuentre la comunicación (4, 9, 13, 16, 17). Las fístulas Barrow A predominan en pacientes hombres, jóvenes y están asociadas en un $80-90 \%$ a trau-matismos craneoencefálicos (13). Usualmente ocurre una ruptura de la pared de la $\mathrm{ACl}$ en su porción cavernosa, provocando así un alto flujo hacia el seno cavernoso.

Estas no suelen resolver espontáneamente y deben de tratarse pronto para evitar le-siones permanentes a estructuras adyacentes. Las fístulas indirectas tipo $B$ son aque-llas que surgen de las ramas intracavernosas de la $\mathrm{ACl}$ hacia el seno cavernoso, las tipo $C$ involucran ramas de la arteria carótida externa y las tipo $D$ incluyen ramas me-níngeas tanto de la carótida externa como de la $\mathrm{ACl}(3,4)$. Las indirectas característicamente suelen ser de bajo flujo y se originan por condiciones que predisponen a debilidad de la pared media de la arteria. Comúnmente se han descrito hipertensión, embarazo, climaterio, trombosis del seno cavernoso, colagenopatías (ej. Ehler-Danlos), aneurismas o pseudoaneurismas. $(3,13)$

El diagnóstico de una fístula puede ser difícil si no se conoce su presentación. El cuadro es variado y va a depender de distintos factores, incluyendo la localización, el tamaño, la ruta del drenaje venoso y la presencia de circulación colateral $(1,18)$. El inicio de las manifestaciones clínicas es insidioso y progresivo. Los signos oftalmoló- gicos son secundarios a la afectación del drenaje venoso anterior hacia la vena of-tálmica. Los más frecuentemente encontrados son: proptosis $(90 \%)$, quemosis (90\%), diplopía (50\%), disminución de la agudeza visual ( $50 \%$ por oclusión de la arteria central de la retina) y soplo orbitario y/o retroauricular $(25 \%)(6)$. Otras manifestaciones incluyen: cefalea, epistaxis, otorragia, hemorragia subaracnoidea o intracraneal, au-mento de la presión intraocular, midriasis, y retinopatía $(1,6,18)$. Clásicamente se ha descrito la triada de Dandy, compuesta por soplo intracraneal (documentado por ul-trasonido), proptosis y quemosis conjuntival, sin embargo su presencia en los pacien-tes es poco frecuente. (1, $6,13,14,19,20)$

Los principales diagnósticos diferenciales a considerar son: trombosis del seno cavernoso, síndrome de fisura orbitaria superior, hematoma retrobulbar, orbitopatía tiroidea, escleritis, tumores tanto benignos como malignos, metástasis, y cualquier otra patología que pueda provocar los signos y síntomas anteriormente descritos. $(3,18$, 21)

El "gold standard" para hacer el diagnóstico de la FCC es la angiografía cere-bral $(2,10,13$, $18,22)$, pues demuestra de forma dinámica el llenado arterial, eviden-ciando la comunicación arteriovenosa y la arterialización de las venas (1).

Hay cinco aspectos que deben analizarse al realizar la arteriografía. Primero, confirmar la bifurcación de la arteria carótida común y el origen de la $\mathrm{ACl}$. Enseguida se debe ubicar la fístula para determinar si es de alto o bajo flujo y si la comunicación es total o parcial. Si el paciente no presenta déficit neurológico contralateral al haber una fístula de alto flujo, se deduce que tiene una circulación colateral que logra com-pensar la irrigación a través de la carótida interna hacia el polígono de Willis. Si no se logra determinar la morfología de la fístula con los marcos usuales de la arteriografía, la maniobra de Mehringer- 
Hieshima o un balón de doble lumen pueden resultar úti-les. (23). El tercer aspecto a evaluar es la permeabilidad de la arteria comunicante anterior al comprimir la $\mathrm{ACl}$ contralateral y posteriormente mediante la maniobra de Heuber se corrobora la permeabilidad de la arteria comunicante posterior. Por último, se debe valorar el drenaje venoso con el fin de descartar una emergencia médica provocada por un defecto en el drenaje cortical. (4)

Otros métodos de imagen que pueden apoyar el diagnóstico incluyen el angio-TAC y la RM, la cual excepcionalmente podría mostrar una hipertrofia de los músculos extraoculares y dilatación venosa. No obstante, su utilidad se limita a descartar otros diagnósticos. $(4,5,9,23,24)$

El tratamiento definitivo de elección en las FCCs directas es endovascular, ya sea transarterial o transvenoso, puesto que tiene bajo riesgo de complicaciones y una mortalidad menor al $1 \%$. Más del $80 \%$ de los pacientes que se sometan a terapia de endovascular presentarán resolución completa de sus síntomas (11). Existen diferentes métodos para lograr el cierre de la fístula, tales como embolización arterial me-diante balón o un coil, embolización química con acrílico o alcohol, o la colocación de un stent (3). Previo al procedimiento se debe comprobar el flujo colateral adecuado de la $\mathrm{ACl}$ hacia el polígono de Willis. Si se emboliza sin circulación alternativa, habría ausencia de flujo al hemisferio cerebral ipsilateral. $(1,9,10,11,12,14,15,18,25,26,27)$

Las complicaciones tras el abordaje endovascular son infrecuentes, excepto en pacientes con colagenopatías (9). Entre las descritas están la hemorragia en el sitio de entrada, perforación de órbita, infección local, déficits neurológicos permanentes o transitorios, dolor facial, diabetes insípida, hemorragia intracerebral, y disminución de la agudeza visual, $(9,11)$.

\section{Conclusión}

La FCC puede ser una causa importante de morbilidad, particularmente ocular. Sus manifestaciones son muy variadas y poco específicas, por lo que suele subdiag-nosticarse debido a la falta de sospecha. Es importante recordar que una correcta identificación de la patología permite tratar la fístula rápidamente. Por este motivo, se recomienda conocer la presentación clínica, realizar un pronto diagnóstico e instaurar la terapia endovascular para evitar complicaciones o secuelas a largo plazo.

La importancia de reportar casos como éste, yace en documentar y compartir el abordaje brindado. Al tener una base de evidencia y contribuir a estandarizar el debi-do manejo y tratamiento se podrá crear un eventual protocolo a seguir.

\section{ANEXOS}

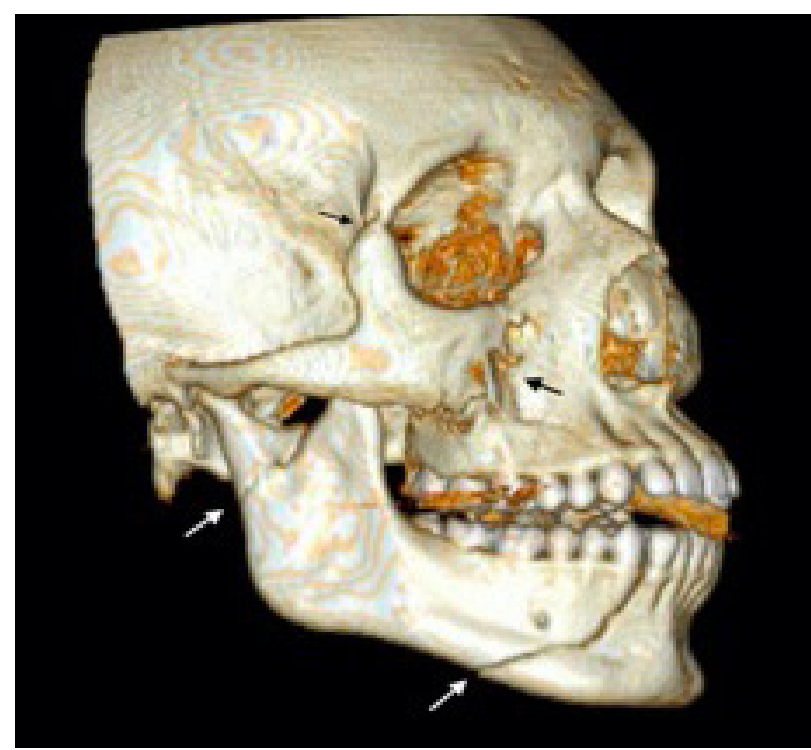

Figura 1: Reconstrucción 3-D, TAC de macizo facial. Flechas blancas: fractura para-sinfisiaria mandibular derecha y fractura de cóndilo mandibular derecho. Flecha ne-gra: fractura malar derecha y de arco cigomático derecho. 


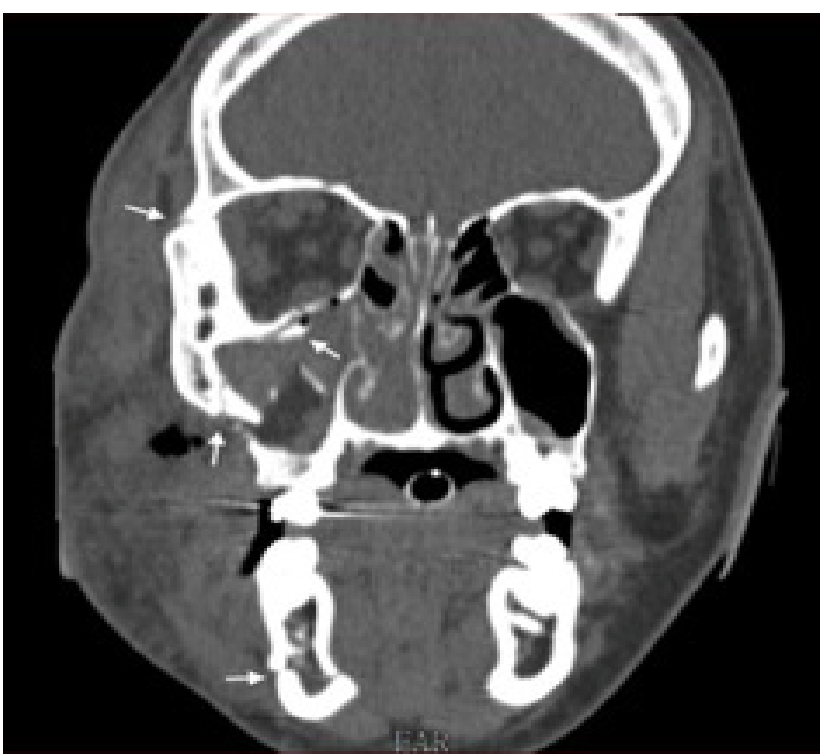

Figura 2: Corte coronal de TAC con macizo facial. Flechas blancas muestran fractura parasinfisiaria mandibular derecha y fractura de cóndilo mandibular derecho y fractu-ra malar derecha y de arco cigomático derecho.

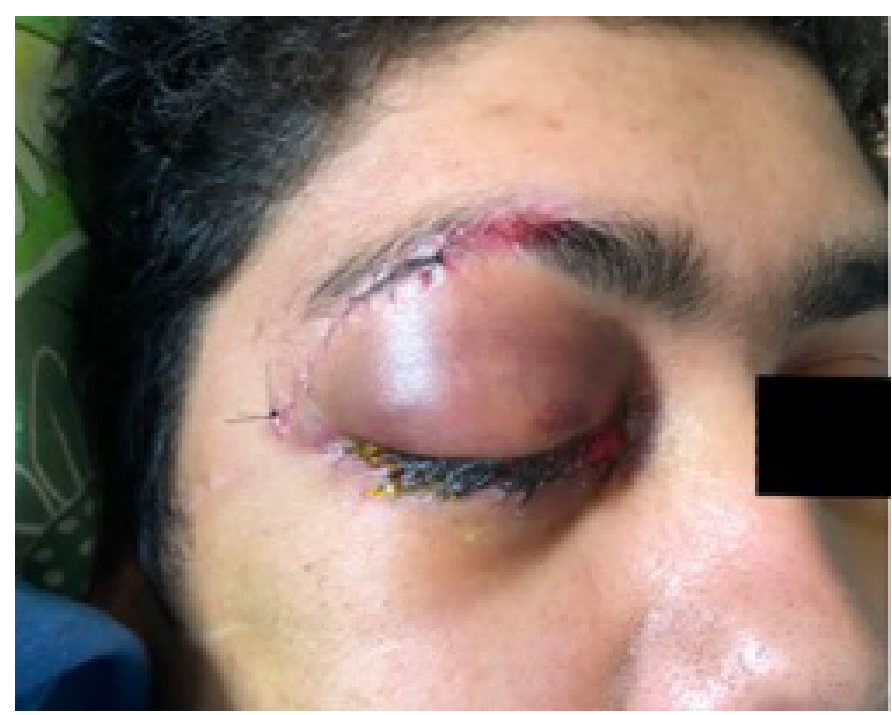

Figura 3: Edema palpebral y quemosis en ojo derecho previo a diagnóstico de FCC postoperatorio día 7.

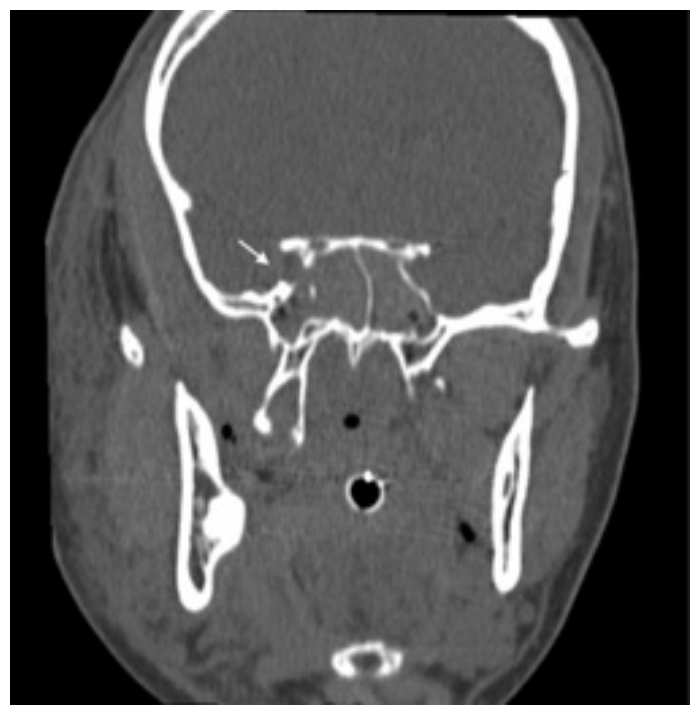

Figura 4: Corte coronal de TAC con macizo facial. Flecha blanca muestra fractura de hueso esfenoides en la pared lateral del seno cavernoso derecho.

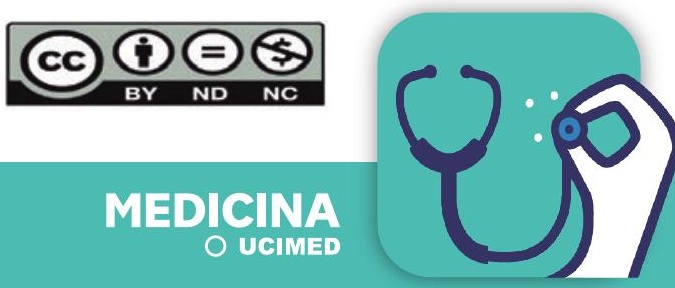




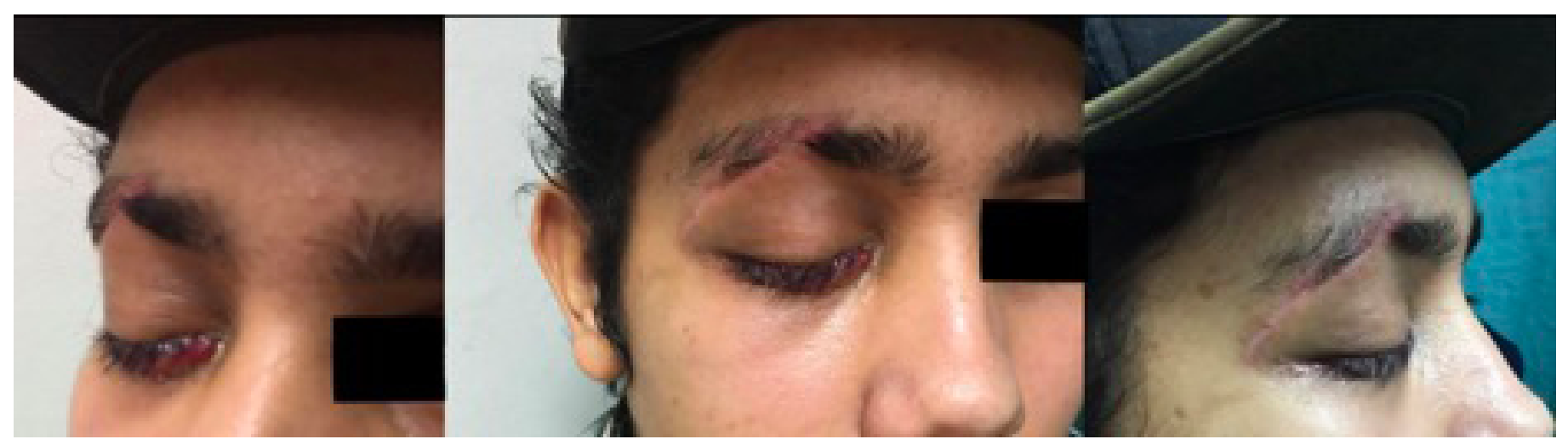

Figura 5: Disminución de edema, quemosis y proptosis dos meses después de reso-lución quirúrgica.

\section{Bibliografía}

1.Calle $\mathrm{MI}$, Hernández $\mathrm{OH}$. Fístula carótidocavernosa espontánea bilateral: re-porte de caso y revisión de la literatura. Acta Colomb Cuid Intensivo. 2016;16(1):23-30.

2.Lang M, Habboub G, Mullin JP, Rasmussen PA. A brief history of carotid-cavernous fistula. J Neurosurg. 2017;126(6):1995-2001.

3.Henderson AD, Miller NR. Carotid-cavernous fistula: current concepts in aetiol-ogy, investigation, and management. Eye. 2018; 32: 164-172.

4.Ringer AJ, Salud L, Tomsick TA. Carotid Cavernous Fistulas: Anatomy, Classifi-cation, and Treatment. Neurosurg Clin N Am. 2005; 16: 279295.

5.Docherty G, Eslami M, Jiang K, Barton J.S. Bilateral carotid cavernous sinus fis-tula: a case report and review of the literature. J Neurol. 2018; 265: 453-459.

6.De Dompablo E, Díez-Álvarez L, Ruíz-Casas D, Sánchez-Gútierrez, Ciancas E, Gonzalez-López J.J. Desprendimiento neurosensorial macular recurrente en fístula carótido-cavernosa. Arch Soc Esp Oftalmol. 2015; 90(7):331-334.

7.Lang-Serrano G. Fistulas carótido cavernosa. Med leg Costa Rica. 2014; 31(1): 135-139.

8.Torres R. Tratamiento endovascular de fístulas carotídeo-cavernosas traumáti-cas: experiencia preliminar en Costa. Rica. Neuroeje. 2005; 19:1723

9.Miller, NR. Dural Carotid-Cavernous Fistulas: Epidemiology, Clinical Presenta-tion, and Management. Neurosurg Clin N Am. 2012; 23: 179-192.

10.Cossu G, Al-Taha K, Hajdu SD, Daniel RT, Messerer M. Carotid-Cavernous Fis-tula After Transsphenoidal Surgery: A Rare but Challenging Complication. World Neurosurg. 2020; 134:221227.

11.Ellis J, Goldstein H, Conolly S, Meyes PM. Carotid-cavernous fistulas. Neuro-surg Focus. 2012; 32(5): E9.

12.Che Ani MF, Kumar R, Md Noh MSF, Muda AS. Supraclinoid direct carotid-cavernous sinus fistula. BJR Case Rep. 2018; 4: 20170058. 
13.Sánchez ME, Jordán JA, Montesinos I, Figueredo J. Fístula carótido-cavernosa postraumática. Presentación de un caso. Invest Medicoquir. 2016; 8(1):135-47.

14.Otiniano R, Vélez M, Flores M, Torres L, Solís F. Congestión venosa en tronco encefálico debido a una fístula carótido-cavernosa directa: a propósito de un caso. Neurocirugía. 2019; 31: 98-102.

15.Genmete JJ, Ansari SA, Gandhi DM. Endovascular Techniques for Treatment of Carotid-Cavernous Fistula. J Neuro-Ophthalmol. 2009; 29: 62-71.

16. Naragum V, Barest G, AbdalKader M, Cronk K, Nguyen TN. Spontaneous Reso-lution of PostTraumatic Direct Carotid-Cavernous Fistula. Intervent Neurol. 2018; 7: 1-5.

17. Sur S, Menaker SA, Alvarez C, et al. Multimodal Management of Carotid-Cavernous Fistulas. World Neurosurg. 2020; 133: e796-e803.

18. Torres M, Rubio W, Conde G, et al. Síntomas neurológicos de fístula carotídeo cavernosa: reporte de caso. Acta Neurol Colomb. 2017; 33(4): 274-278.

19. Fuentes D, Cáceres YA, Hodelín D. Importancia del método clínico en el diag-nóstico de la fístula carótido-cavernosa. Medisan 2016; 20(12): 25192525.

20. Albuquerque Morais B, Yakai $\mathrm{Vn}$, Mendes Pereira Caldas JG, Silva Paiva W, Matushita $H$, Jacobsen Texeira M. Post-traumatic carotidcavernous fistula in a pediatric patient: a casebased literature review. Childs Nerv Syst. 2018; 34: 577-580.

21. Wang D, Sun ZM, Wu J. A rare case report of complications in craniofacial inju-ries - Cavernous sinus-carotid fistula. Medicine. 2017; 96: 52(e9511).

22. Bailey CR, Ray-Mazumder N, Manesh RS. Carotid Cavernous Fistula. J Gen Intern Med. 2016; 32(4):483-4.

23. De Keizer, RJW. Carotid-cavernous and orbital arteriovenous fistulas: ocular features, diagnostic and hemodynamic considerations in relation to visual im-pairment and morbidity. Orbit. 2003; 22:

121-142

24. Zanaty M, Chalouhi N, Tjoumakaris SI, Hasan $\mathrm{D}$, Rosenwasser RH, Jabbour P.. Endovascular Treatment of Carotid-Cavernous Fistulas. Neurosurg Clin N Am. 2014; 25(3):551-563.

25. Ertl L, Brückmann H, Patzig M, Fesl G. Endovascular therapy of direct dural ca-rotid cavernous fistulas - A therapy assessment study including long-term fol-low-up patients interviews. PLoSONE 2019; 14(10): e0223488.

26. Nossek E, Lombardo K, Schneider JR, Kwan K, Chalif DJ, Setton A. Unilateral Venous Approach to Contralateral or Bilateral Carotid Cavernous Shunts. World Neurosurg. 2020; 133: e479-e486.

27. Niu Y, Chen T, Tang J, Jiang ZY, Zhu G, Chen Z. Detachable balloon emboliza-tion as the preferred treatment option for traumatic carotid-cavernous sinus fistu-la? Interv Neuroradiol. 2020; 26(1):90 